IFUP-TH/2013-03

LAPTH-003/13

\title{
Three-manifold invariant from functional integration
}

\author{
Enore Guadagnini ${ }^{a}$ and Frank Thuillier ${ }^{b}$ \\ a Dipartimento di Fisica "E. Fermi" dell'Università di Pisa and INFN, Sezione di Pisa, Italy. \\ ${ }^{b}$ LAPTH, Chemin de Bellevue, BP 110, F-74941 Annecy-le-Vieux cedex, France.
}

\begin{abstract}
We give a precise definition and produce a path-integral computation of the normalized partition function of the abelian $U(1)$ Chern-Simons field theory defined in a general closed oriented 3-manifold. We use the Deligne-Beilinson formalism, we sum over the inequivalent $U(1)$ principal bundles over the manifold and, for each bundle, we integrate over the gauge orbits of the associated connection 1forms. The result of the functional integration is compared with the abelian $U(1)$ Reshetikhin-Turaev surgery invariant.
\end{abstract}




\section{Introduction}

Gauge quantum field theories play a fundamental role in the description of physical phenomena. Most of the models that have been considered so far are defined in Minkowski space. But one can imagine that, in certain conditions, it will become important to study a quantum gauge theory defined in a topological nontrivial manifold. In this paper we will consider a quantum field theory with a $U(1)$ local gauge symmetry which is defined in a general connected closed oriented 3-manifold $M$; the action is given by the Chern-Simons functional and the observables of this model represent topological invariants $[1,2,3,4,5,6]$.

In the present article we shall concentrate on the quantum field theory aspects which are related with the path-integral definition and with the computation of the normalized partition function of the theory, which represents a topological invariant of the 3-manifold $M$. By using the Deligne-Beilinson formalism, it turns out that the result of the functional integration for the normalized partition function of the $\mathrm{U}(1)$ Chern-Simons theory is strictly related with the Reshetikhin-Turaev $U(1)$ surgery invariants of 3-manifolds $[7,8,9]$.

\subsection{Summary and results}

Let us give a short description of the content of our paper and a presentation of the main results. In the Deligne-Beilinson (DB) formalism $[5,10,11,12,13]$, each gauge orbit $A$ of a $U(1)$ connection on the 3 -manifold $M$ is a class belonging to the DB cohomology space $H_{D}^{1}(M)$. The Chern-Simons action $S$ is given [5] by

$$
S[A]=2 \pi k \int_{M} A * A
$$

where the $*$-product denotes the pairing $H_{D}^{1}(M) \times H_{D}^{1}(M) \rightarrow H_{D}^{3}(M) \sim \mathbb{R} / \mathbb{Z}$ which is associated with the canonical DB product [13]. A modification of the orientation of the manifold $M$ is equivalent to a change in the sign of the integer coupling constant $k$, so one can choose $k>0$. Let $\Omega^{1}(M)$ be the space of the 1 -forms on $M$ and $\Omega_{\mathbb{Z}}^{1}(M)$ the subspace of closed forms with integral periods. The space $\Omega_{\mathbb{Z}}^{1}(M)$ corresponds to the set of gauge transformations. A presentation of $H_{D}^{1}(M)$ is given $[10,11,12,13,14]$ by the following exact sequence

$$
0 \rightarrow \Omega^{1}(M) / \Omega_{\mathbb{Z}}^{1}(M) \rightarrow H_{D}^{1}(M) \rightarrow H^{2}(M) \rightarrow 0,
$$

in which $H^{2}(M)$ denotes the second integral cohomology group of $M$ and, because of Poincaré duality, $H^{2}(M) \simeq H_{1}(M)$ where $H_{1}(M)$ stands for the first homology group of $M$. Thus

$H_{D}^{1}(M)$ can be understood as an affine bundle over $H_{1}(M)$ for which $\Omega^{1}(M) / \Omega_{\mathbb{Z}}^{1}(M)$ acts as a translation group on the fibres. More precisely, each fibre is characterized by an element 
of $H_{1}(M)$; a generic DB class $A$ that belongs to the fibre over $\gamma \in H_{1}(M)$ can be written as

$$
A=\widehat{A}_{\gamma}+\omega
$$

where $\omega \in \Omega^{1}(M) / \Omega_{\mathbb{Z}}^{1}(M)$. The element $\widehat{A}_{\gamma}$ just fixes an origin on the fibre over $\gamma$ and any other element of this fibre can be obtained from $\widehat{A}_{\gamma}$ by means of a translation with the 1-form $\omega$ modulo closed forms of integral periods. For each fibre, the choice of the corresponding origin class $\widehat{A}_{\gamma}$ is not unique. On the fibre over the trivial element of $H_{1}(M)$ one can take as canonical origin the zero class, $\widehat{A}_{0}=0$, which is precisely the gauge orbit of the vanishing connection.

Each DB class $A \in H_{D}^{1}(M)$ describes a $U(1)$ principal bundle with connection (up to gauge transformations), and equation (1.2) shows that the inequivalent principal $U(1)$ bundles can be labelled by $H_{1}(M)$. Let us assume [5] that the functional integration consists of a sum over the inequivalent principal bundles and, for each bundle, of a sum over the gauge orbits of the corresponding connection 1-forms. According to equation (1.3), this means that the path-integral is given by $\int D A e^{i S[A]}=\sum_{\gamma \in H_{1}(M)} \int D \omega e^{i S\left[\widehat{A}_{\gamma}+\omega\right]}$ where one has a sum over all the elements of the homology group of the manifold.

Let us define the normalized partition function $Z_{k}(M)$ as

$$
Z_{k}(M)=\frac{\sum_{\gamma \in H_{1}(M)} \int D \omega e^{i S\left[\widehat{A}_{\gamma}+\omega\right]}}{\int D \omega e^{i S[\omega]}} .
$$

The normalization factor $\int D \omega e^{i S[\omega]}=\int D \omega e^{i S\left[\widehat{A}_{0}+\omega\right]}$ just corresponds to the functional integral associated with the fibre of $H_{D}^{1}(M)$ over the trivial element $0 \in H_{1}(M)$; i.e. $\int D \omega e^{i S[\omega]}$ represents the integral over the gauge orbits of the connection 1-forms of the trivial principal bundle over $M$.

Remark 1.1. In quantum field theories one is really concerned with distributional fields, so one may be interested in the possible modifications of sequence (1.2) under rough extensions of the fields space. Quite remarkably, the basic structure of the configuration space - as described by sequence (1.2) - is stable under the inclusion of distributional configurations. Indeed there is a natural inclusion $[5,6]$ of $H_{D}^{1}(M)$ and of the space $Z_{1}(M)$ of 1-cycles in $M$ into the Pontrjagyn dual $\operatorname{Hom}\left(H_{D}^{1}(M), S^{1}\right)$ of $H_{D}^{1}(M)$. These inclusions are ensured by the canonical DB product and the $\mathbb{R} / \mathbb{Z}$-valued integration over 1-cycles of $M$. This dual space contains generalized (i.e. distributional) connections and it is embedded into the exact sequence

$$
0 \rightarrow \operatorname{Hom}\left(\Omega_{\mathbb{Z}}^{2}(M), S^{1}\right) \rightarrow \operatorname{Hom}\left(H_{D}^{1}(M), S^{1}\right) \rightarrow H^{2}(M) \rightarrow 0 .
$$

Let us introduce the simplified notation

$$
H_{D}^{1}(M)^{*} \equiv \operatorname{Hom}\left(H_{D}^{1}(M), S^{1}\right) \quad, \quad \Omega_{\mathbb{Z}}^{2}(M)^{*} \equiv \operatorname{Hom}\left(\Omega_{\mathbb{Z}}^{2}(M), S^{1}\right)
$$


note that there also is a natural inclusion

$$
\frac{\Omega^{1}(M)}{\Omega_{\mathbb{Z}}^{1}(M)} \hookrightarrow \Omega_{\mathbb{Z}}^{2}(M)^{*} .
$$

Equation (1.3) admits a distributional extension in which $\widehat{A}_{\gamma} \in H_{D}^{1}(M)^{*}$ and $\omega \in \Omega_{\mathbb{Z}}^{2}(M)^{*}$.

In general, the abelian homology group $H_{1}(M)$ can be decomposed as $H_{1}(M)=F(M) \oplus$ $T(M)$, where $F(M)$ is freely generated and the torsion component $T(M)$ can be written as a direct sum of $\mathbb{Z}_{p} \equiv \mathbb{Z} / p \mathbb{Z}$ factors. For torsion-free manifolds, when the torsion component $T(M)$ is trivial, the main properties of the path-integral have been studied in Ref.[5]. In the present article we shall concentrate on the pure torsion case, in which the freely generated component $F(M)$ is trivial and then $H_{1}(M)$ is a finite group

$$
H_{1}(M)=T(M)=\mathbb{Z}_{p_{1}} \oplus \mathbb{Z}_{p_{2}} \oplus \cdots \oplus \mathbb{Z}_{p_{w}},
$$

in which the torsion numbers $\left\{p_{1}, p_{2}, \ldots, p_{w}\right\}$ are fixed by the convention that $p_{i}$ divides $p_{i+1}$. Some preliminary results on the pure torsion case have been discussed in Ref.[6]. The action (1.1) is a quadratic function of the fields and then the result of the functional integration (1.4) does not depend on the particular choice of the origin class $\widehat{A}_{\gamma}$ for each $\gamma \in H_{1}(M)$.

Proposition 1. For each torsion element $\gamma \in T(M)$, one can select the origin class $\widehat{A}_{\gamma}$ to correspond to a stationary point of the action, i.e. $\widehat{A}_{\gamma}$ can be chosen to be equal to the gauge orbit $A_{\gamma}^{0}$ of a flat connection. Therefore the normalized partition function (1.4) can be written as a sum over the gauge orbits of flat connections

$$
Z_{k}(M)=\frac{\sum_{\gamma \in H_{1}(M)} \int D \omega e^{i S\left[A_{\gamma}^{0}+\omega\right]}}{\int D \omega e^{i S[\omega]}}=\sum_{\gamma \in H_{1}(M)} e^{i S\left[A_{\gamma}^{0}\right]} .
$$

Indeed, $S\left[A_{\gamma}^{0}+\omega\right]=S\left[A_{\gamma}^{0}\right]+S[\omega]+2 \pi k \int A_{\gamma}^{0} * \omega$ but since $A_{\gamma}^{0}$ is the class of a flat connection and $\omega$ is globally well defined in $M$, the last term is vanishing and therefore $S\left[A_{\gamma}^{0}+\omega\right]=S\left[A_{\gamma}^{0}\right]+S[\omega]$. Consequently

$$
\begin{aligned}
Z_{k}(M) & =\frac{\sum_{\gamma \in H_{1}(M)} \int D \omega e^{i S\left[A_{\gamma}^{0}\right]} e^{i S[\omega]}}{\int D \omega e^{i S[\omega]}}= \\
& =\sum_{\gamma \in H_{1}(M)} e^{i S\left[A_{\gamma}^{0}\right]} \frac{\int D \omega e^{i S[\omega]}}{\int D \omega e^{i S[\omega]}}=\sum_{\gamma \in H_{1}(M)} e^{i S\left[A_{\gamma}^{0}\right]} .
\end{aligned}
$$

On the other hand, since the value of the path-integral does not depend on the choice of the origins in $H_{D}^{1}(M)^{*}$, for each $\gamma$ one finds

$$
e^{i S\left[A_{\gamma}^{0}\right]}=\frac{\int D \omega e^{i S\left[\widehat{A}_{\gamma}+\omega\right]}}{\int D \omega e^{i S[\omega]}} .
$$


If $\widehat{A}_{\gamma}$ satisfies $S\left[\widehat{A}_{\gamma}\right]=0 \bmod \mathbb{Z}$, then

$$
e^{i S\left[A_{\gamma}^{0}\right]}=\frac{\int D \omega e^{i S[\omega]} e^{4 \pi i k \int \omega * \widehat{A}_{\gamma}}}{\int D \omega e^{i S[\omega]}},
$$

and by means of the path-integral (1.12) one can compute the amplitude $e^{i S\left[A_{\gamma}^{0}\right]}$.

Let us introduce a set of generators $\left\{h_{1}, h_{2}, \ldots, h_{w}\right\}$ for $H_{1}(M)$; the element $h_{i}$ is a generator for $\mathbb{Z}_{p_{i}}$, with $p_{i} h_{i}=0$. A generic element $\gamma \in H_{1}(M)$ can be described by means of the sum $\gamma=\sum_{i=1}^{w} n_{i} h_{i}$ with integers $\left\{n_{i}\right\}$. Each term $e^{i S\left[A_{\gamma}^{0}\right]}$ can now be written as

$$
e^{i S\left[A_{\gamma}^{0}\right]}=e^{2 \pi i k \sum_{i j} n_{i} n_{j} Q_{i j}}
$$

where the matrix $Q_{i j}$ determines a $\mathbb{Q} / \mathbb{Z}$-valued quadratic form $Q$ on the torsion group $T(M)$. Although $Q$ only depends $[16,17,18]$ on the manifold $M$, in order to describe the result of the functional integration (1.12), it is useful to consider a surgery presentation [19] of $M$ in $S^{3}$.

Let $\mathcal{L}=\mathcal{L}_{1} \cup \mathcal{L}_{2} \cdots \cup \mathcal{L}_{m} \subset S^{3}$ be a framed surgery link — associated with a Dehn surgery presentation of $M$ in $S^{3}$ - with integer surgery coefficients and let $\mathbb{L}$ denote the corresponding linking matrix. When the homology group is given by equation (1.8), one can always find a surgery presentation in which the linking matrix $\mathbb{L}$ is non-degenerate (invertible), so we assume that this is indeed the case. For each link component $\mathcal{L}_{t}$ (with $t=1,2, \ldots, m$ ), let $G_{t}$ be a simple small circle linked with $\mathcal{L}_{t}$ which can be taken as a generator of the homology of the complement of $\mathcal{L}_{t}$ in $S^{3}$; then $\left\{G_{1}, \ldots, G_{m}\right\}$ is a set of generators for the homology of $S^{3}-\mathcal{L}$. The homology group $H_{1}(M)$ admits the presentation

$$
H_{1}(M)=\left\langle G_{1}, \ldots, G_{m} \mid\left[\mathcal{L}_{1 \mathrm{f}}\right]=0,\left[\mathcal{L}_{2 \mathrm{f}}\right]=0, \ldots,\left[\mathcal{L}_{m \mathrm{f}}\right]=0\right\rangle
$$

where $\left[\mathcal{L}_{t \mathrm{f}}\right]$ is the homology class $\left(\right.$ in $S^{3}-\mathcal{L}$ ) of the framing $\mathcal{L}_{t \mathrm{f}}$ of the component $\mathcal{L}_{t}$

$$
\left[\mathcal{L}_{t \mathrm{f}}\right]=\sum_{s=1}^{m} \mathbb{L}_{t s} G_{s}
$$

Each generator $h_{i}$ of $H_{1}(M)$ can be written as a linear combination of the $\left\{G_{t}\right\}$ generators

$$
h_{i}=\sum_{t=1}^{m} B_{i t} G_{t} \quad, \quad(i=1,2 \ldots, w)
$$

with integer coefficients $B_{i t}$. 
Corollary 1. In the basis defined by the generators $\left\{h_{i}\right\}$, the matrix elements $Q_{i j}$ of the quadratic form on the torsion group $T(M)$ are given by

$$
Q_{i j}=\sum_{t, s=1}^{m} B_{i t} B_{j s} \mathbb{L}_{t s}^{-1},
$$

where $\mathbb{L}^{-1}$ represents the inverse in $\mathbb{R}^{m}$ of the linking matrix; the normalized partition function $Z_{k}(M)$ takes the form

$$
Z_{k}(M)=\sum_{n_{1}=0}^{p_{1}-1} \sum_{n_{2}=0}^{p_{2}-1} \cdots \sum_{n_{w}=0}^{p_{w}-1} e^{2 \pi i k \sum_{i j} n_{i} n_{j} Q_{i j}} .
$$

Moreover,

$$
Z_{k}(M)=\left(p_{1} p_{2} \cdots p_{w}\right)^{1 / 2} I_{k}(M),
$$

where $I_{k}(M)$ denotes the value of the Reshetikhin-Turaev U(1) surgery invariant of the 3manifold $M$.

As a matter of facts, in the definition and in the computation of the normalized partition function $Z_{k}(M)$ of the $U(1)$ Chern-Simons theory there is no need of introducing a metric in the 3-manifold $M$; moreover, $Z_{k}(M)$ has nothing to do with the perturbative gauge-fixing procedure.

The paper is organized as follows. The basic rules which are used in the computation of the field theory path-integrals are listed in Section 2. Section 3 contains a proof of Proposition 1 and Corollary 1 together with a path-integral derivation of expression (1.17) of the normalized partition function. One illustrative example is presented in Section 4.

\section{Computation rules}

In certain expressions of the previous section, ratios of functional integrations - as indicated for instance in equations (1.4) and (1.12) - appear. This notation belongs to the set of standard conventions which are employed in physics, in which any meaningful quantity takes the form of a ratio of regularized functional integrations in the limit in which the regularization is removed.

Remark 2.1. Each functional integration, which formally involves an infinite number of integration variables, can be approximated or regularized by restricting the integral to a finite number $N$ of variables; this regularization is removed in the $N \rightarrow \infty$ limit. The ratio of two path-integrals means: (1) introduce a regularization in the numerator and in the 
denominator simultaneously (with the same finite $N$ ), (2) for each fixed $N$, the regularized ratio - that is the ratio of the two regularized integrals - is well defined and depends on $N$, (3) finally consider the $N \rightarrow \infty$ limit of the regularized ratio. For the ratios of functional integrations considered in quantum field theory, this limit normally exists. For example, all the perturbative computations in quantum electrodynamics or in the $S U(3)_{c} \times S U(2)_{L} \times$ $U(1)_{Y}$ Standard Model of the particles interactions are based precisely on the existence of this limit for the appropriate ratios of functional integrations. In any path-integral expression one must specify the so-called overall normalization, i.e. the choice of the functional integration which appears in the denominator, because different normalizations generally give rise to different results. The path-integrals in which the normalization is not specified are not well defined.

The limit procedure which has been mentioned in Remark 2.1 ensures the validity of the following two properties.

(P1) Linearity. If, in a given quantum field theory, the functional integration region $R$ is the union of two disjoint parts, $R=R_{1} \cup R_{2}$, then the path-integral over $R$ is the sum of the path-integrals over $R_{1}$ and over $R_{2}$.

(P2) Translation invariance. Suppose that, in a given quantum field theory, any field configuration $\phi(x)$ can be written as

$$
\phi(x)=\phi_{0}(x)+\psi(x)
$$

where $\phi_{0}(x)$ is fixed and the variable $\psi(x)$ can fluctuate. When the action $S[\phi]$ is a quadratic function of the field variables, the functional integration is invariant [20] under translation

$$
\langle X(\phi)\rangle \equiv \frac{\int D \phi e^{i S[\phi]} X(\phi)}{\int D \phi e^{i S[\phi]}}=\frac{\int D \psi e^{i S\left[\phi_{0}+\psi\right]} X\left(\phi_{0}+\psi\right)}{\int D \phi e^{i S[\phi]}} .
$$

The basic properties (P1) and (P2) can also be understood as defining relations because all our functional integral computations are based precisely on these two properties exclusively. For instance, properties (P1) and (P2) have been used to write equation (1.4).

Each gauge orbit $A$ can be represented by a field configuration which admits a Čech-de Rham representation, i.e. a representative of the class $A$ can be described by a collection of local variables which, in a good covering $\left\{\mathcal{U}_{a}\right\}$ of $M$, are given by

$$
A \leftrightarrow\left(v^{a}, \lambda^{a b}, n^{a b c}\right)
$$

$v^{a}$ denotes a 1 -form locally defined in the open set $\mathcal{U}_{a} ; \lambda^{a b}$ represents a 0 -form in the intersection $\mathcal{U}_{a} \cap \mathcal{U}_{b}$ such that $v^{a}-v^{b}=d \lambda^{a b}$, and the integer $n^{a b c}$ is defined in $\mathcal{U}_{a} \cap \mathcal{U}_{b} \cap \mathcal{U}_{c}$ with 
the property $\lambda^{a b}+\lambda^{b c}+\lambda^{c a}=n^{a b c}$. In our notations, a particular representative element of the DB class, which appears on the left-hand-side of the arrow $\leftrightarrow$, is described by the collection of Cech-de Rham field components that are shown on the right-hand-side of $\leftrightarrow$. In particular a representative of a class $\omega \in \Omega^{1}(M) / \Omega_{\mathbb{Z}}^{1}(M)$ fulfills

$$
\omega \leftrightarrow\left(\omega^{a}, 0,0\right),
$$

where $\left\{\omega^{a}\right\}$ are the restrictions in the open sets $\left\{\mathcal{U}_{a}\right\}$ of a 1-form on $M$ also denoted by $\omega$. Vice versa, given a 1-form $\alpha \in \Omega^{1}(M)$, the DB class associated with $\alpha$ has Čech-de Rham representation

$$
\alpha \leftrightarrow\left(\alpha^{a}, 0,0\right),
$$

where $\left\{\alpha^{a}\right\}$ are the restrictions of $\alpha \in \Omega^{1}(M)$ in the open sets $\left\{\mathcal{U}_{a}\right\}$. Note that if $\omega \in$ $\Omega^{1}(M) / \Omega_{\mathbb{Z}}^{1}(M)$ then $\int \omega * \omega=\int \omega \wedge d \omega \bmod \mathbb{Z}$.

In the $U(1)$ Chern-Simons field theory, a typical path-integral computation - that will appear below - takes the form

$$
\left\langle\left\langle e^{2 \pi i \int \omega * \alpha}\right\rangle\right\rangle \equiv \frac{\int D \omega e^{2 \pi i k \int \omega * \omega} e^{2 \pi i \int \omega * \alpha}}{\int D \omega e^{2 \pi i k \int \omega * \omega}},
$$

where $\omega \in \Omega_{\mathbb{Z}}^{2}(M)^{*}$ represents the integration variable, whereas $\alpha$ can be interpreted as a given classical external source. Let us first consider the case in which $\alpha$ is the DB class associated with a 1 -form $\alpha \in \Omega^{1}(M)$. Let $\alpha^{\prime}$ be the DB class such that

$$
\alpha^{\prime} \leftrightarrow\left(\frac{1}{2 k} \alpha^{a}, 0,0\right)
$$

One can put

$$
\omega=-\alpha^{\prime}+\widetilde{\omega},
$$

where $\alpha^{\prime}$ is fixed and the variable $\widetilde{\omega} \in \Omega_{\mathbb{Z}}^{2}(M)^{*}$ can fluctuate. Since $D \omega=D \widetilde{\omega}$ (property $(\mathrm{P} 2))$ and $[5]$

$$
k \int \omega * \omega+\int \omega * \alpha=k \int \widetilde{\omega} * \widetilde{\omega}-k \int \alpha^{\prime} * \alpha^{\prime},
$$

one finds

$$
\left\langle\left\langle e^{2 \pi i \int \omega * \alpha}\right\rangle\right\rangle=e^{-2 \pi i k \int \alpha^{\prime} * \alpha^{\prime}} \frac{\int D \widetilde{\omega} e^{2 \pi i k \int \widetilde{\omega} * \widetilde{\omega}}}{\int D \omega e^{2 \pi i k \int \omega * \omega}}=e^{-2 \pi i k \int \alpha^{\prime} * \alpha^{\prime}} .
$$

Because $\alpha$ is globally defined in $M$, one finally obtains [5]

$$
e^{-2 \pi i k \int \alpha^{\prime} * \alpha^{\prime}}=e^{-(2 \pi i / 4 k) \int \alpha \wedge d \alpha} .
$$


This procedure can also be applied when $\alpha$ is a 1-current. In particular, for each oriented knot $C$ which belongs to a 3 -ball $\mathcal{B}$ inside $M$, one can find a Seifert surface $\Sigma \subset M$ such that $C=\partial \Sigma$. This equation can be written in terms of currents: $j_{C}=d \alpha_{\Sigma}$, where $j_{C}$ is the 2-current of the knot $C$ and $\alpha_{\Sigma}$ is the 1-current of $\Sigma$. The 1-current $\alpha_{\Sigma}$ can be understood as distributional limit of 1-forms in $M$. By construction one has $\int \omega \wedge d \alpha_{\Sigma}=\int \omega \wedge j_{C}=\oint_{C} \omega$.

The DB class $\eta_{C} \in H_{D}^{1}(M)^{*}$ defined by

$$
\eta_{C} \leftrightarrow\left(\alpha_{\Sigma}^{a}, 0,0\right)
$$

only depends on the knot $C$. Then for any $\omega \in \Omega_{\mathbb{Z}}^{2}(M)^{*}$ one has

$$
\int \omega * \eta_{C}=\int \omega \wedge d \alpha_{\Sigma}=\oint_{C} \omega \quad \bmod \mathbb{Z} .
$$

For a two components oriented link $C_{1} \cup C_{2} \subset \mathcal{B} \subset M$, the value of the linking number of $C_{1}$ and $C_{2}$ is given by

$$
\ell k\left(C_{1}, C_{2}\right)=\int \alpha_{\Sigma_{1}} \wedge d \alpha_{\Sigma_{2}}
$$

with $C_{1}=\partial \Sigma_{1}$ and $C_{2}=\partial \Sigma_{2}$. For a single oriented framed knot $C \subset \mathcal{B} \subset M$, the integral $\int \alpha_{\Sigma} \wedge d \alpha_{\Sigma}$ represents the self-linking number of $C=\partial \Sigma$ which is defined to be the linking number of $C$ and its framing $C_{\mathrm{f}}, \int \alpha_{\Sigma} \wedge d \alpha_{\Sigma} \equiv \ell k\left(C, C_{\mathrm{f}}\right)=\int \alpha_{\Sigma} \wedge d \alpha_{\Sigma_{\mathrm{f}}}$.

Consider now equation (2.8) in the case $\alpha=\eta_{L}=\sum_{j} q_{j} \eta_{C_{j}}$ where $\left\{C_{j}\right\}$ are the components of a framed oriented colored link $L=C_{1} \cup \cdots \cup C_{n} \subset \mathcal{B} \subset M$ and $q_{j}$ denotes the color of $C_{j}$; in the DB formalism each color (or charge) $q_{j}$ must assume [5] integer values. One obtains

$$
\left\langle\left\langle e^{2 \pi i \sum_{j=1}^{n} q_{j} \oint_{C_{j}} \omega}\right\rangle\right\rangle=e^{-(2 \pi i / 4 k) \sum_{i j=1}^{n} q_{i} q_{j} \mathbb{L}_{i j}},
$$

where the integers $\mathbb{L}_{i j}$ are the matrix elements of the linking matrix associated with $L$. The result (2.12) can also be obtained by taking $\alpha \in \Omega^{1}(M)$ and considering the $\alpha \rightarrow \eta_{L}$ limit in equation (2.8).

Equation (2.12) also gives the complete solution [5] of the $U(1)$ Chern-Simons quantum field theory defined in $M=S^{3}$ because, in this case, any link belongs to a 3-ball.

The concluding remarks of this section concern some general properties [5] of the expectation values in the abelian Chern-Simons theory.

Remark 2.2. Let the colored oriented and framed link $L^{\prime}=L \cup U \subset M$ be the union of the link $L$ with the unknot $U$. If $U$ belongs to a 3-ball which is disjoint from $L$, and $U$ has trivial framing - i.e. its framing $U_{\mathrm{f}}$ satisfies $\ell k\left(U, U_{\mathrm{f}}\right)=0$ - then the expectation value of the holonomy associated with $L^{\prime}$ is equal to the expectation value of the holonomy associated with $L$. Indeed the expectation value of the holonomy associated with $L^{\prime}$ is the product [5] 
of the expectation values associated with $L$ and with $U$ (this feature can be understood as the topological version of the cluster property of ordinary quantum field theories), and the expectation value of the holonomy associated with the unknot $U$ is equal to the expectation value of the identity.
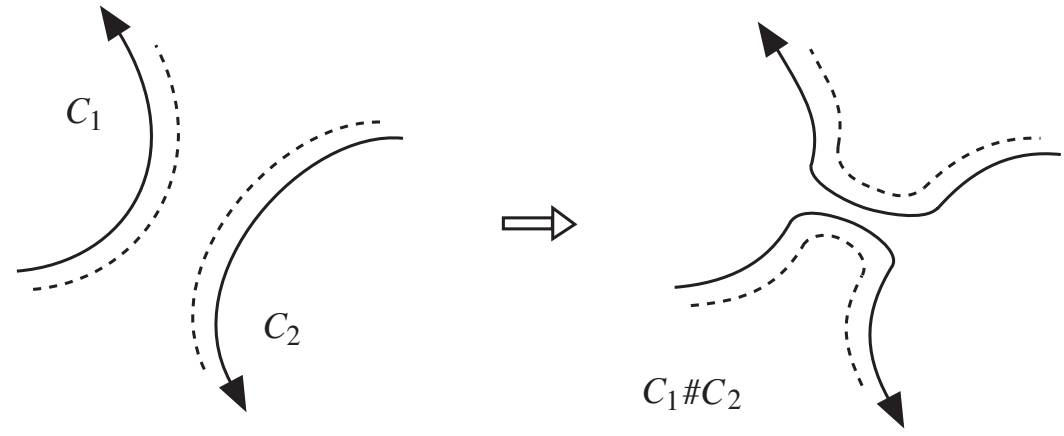

Figure 2.1. Sum of knots.

Definition 2.1. Let $C_{1}$ and $C_{2}$ be two oriented (and possibly framed) knots in $M$. By joining $C_{1}$ and $C_{2}$ in the way shown in Figure 2.1, one obtains the knot $C_{1} \# C_{2}$, that is called the (band connected) sum of $C_{1}$ and $C_{2}$. The dashed lines in Figure 2.1 refer to the framings; by construction, the framing $\left(C_{1} \# C_{2}\right)_{\mathrm{f}}$ of $C_{1} \# C_{2}$ is just the sum of the framings $C_{1 \mathrm{f}} \# C_{2 \mathrm{f}}$. When $M=S^{3}$-which is of particular interest in the Dehn surgery presentation in $S^{3}$ of generic 3-manifold-one has

$$
\ell k\left(\left(C_{1} \# C_{2}\right)_{\mathrm{f}}, C_{1} \# C_{2}\right)=\ell k\left(C_{1 \mathrm{f}}, C_{1}\right)+\ell k\left(C_{2 \mathrm{f}}, C_{2}\right)+2 \ell k\left(C_{1}, C_{2}\right) .
$$

The knot $C_{2}$ with modified orientation is indicated by $-C_{2}$; one finds

$$
\ell k\left(\left(C_{1} \#\left(-C_{2}\right)\right)_{\mathrm{f}}, C_{1} \#\left(-C_{2}\right)\right)=\ell k\left(C_{1 \mathrm{f}}, C_{1}\right)+\ell k\left(C_{2 \mathrm{f}}, C_{2}\right)-2 \ell k\left(C_{1}, C_{2}\right) .
$$

For colored knots $C_{1}$ and $C_{2}$, the sum $C_{1} \# C_{2}$ is well defined when $C_{1}$ and $C_{2}$ have the same color. Since the linking number can be interpreted as an intersection product for which \# plays the role of the standard sum, equations (2.13) and (2.14) also have a natural homological interpretation. The sum of knots enters the Kirby calculus [21].

Remark 2.3. If the knots $C_{1}$ and $C_{2}$ have the same color, the expectation value of the holonomy associated with the link $L=C_{1} \cup C_{2} \cup C_{3} \cup \cdots \cup C_{n}$ is equal to the expectation value of the holonomy associated with $L^{\prime}=C_{1} \# C_{2} \cup C_{3} \cup \cdots \cup C_{n}$. Indeed the expectation values of the link holonomies are invariant under the addition in the link of a (triviallyframed) unknot which belongs to a 3-ball $\mathcal{B} \subset M$ (Remark 2.2); on the other hand, the band 
connected sum of two knots can be obtained by means of the introduction of a triviallyframed unknot - belonging to a 3-ball — which coincides with the boundary of the band (precisely like in the case of the Kirby calculus [21]).

\section{Path-integral partition function}

In this section, the path-integral computation of the normalized partition function will be presented. Following the general method introduced in [5], we first select — as origin $\widehat{A}_{\gamma}$ a distributional class $\eta_{\gamma} \in H_{D}^{1}(M)^{*}$ which is canonically associated with a framed knot that represents $\gamma \in H_{1}(M)$. With this choice of $\widehat{A}_{\gamma}$, the value of the partition function can be expressed by means of a sum of expectation values of knot holomomies.

Similarly to the homology group $H_{1}(M)$ - that can equivalently be computed by using for instance the singular homology or the Čech homology - the quadratic form $Q$ entering equation (1.13) can be determined by using different techniques. The method that we describe is essentially based on knot theory arguments; in Sect.3.1 we also mention an alternative procedure which concerns flat (smooth) connections. Both methods give the same result.

\subsection{Torsion knots and flat connections}

Each element $\gamma \in H_{1}(M)$ can be represented by a oriented knot, denoted by the same symbol $\gamma \subset M$. Let $\eta_{\gamma} \in H_{D}^{1}(M)^{*}$ be the distributional class [5] which is canonically associated with the knot $\gamma \subset M$. Similarly to the case of the gauge orbit of a $U(1)$ gauge field, $\eta_{\gamma}$ admits a Čech-de Rham representation

$$
\eta_{\gamma} \leftrightarrow\left(V_{\gamma}^{a}, \Lambda_{\gamma}^{a b}, N_{\gamma}^{a b c}\right)
$$

in which $d V_{\gamma}^{a}$ is the restriction in $\mathcal{U}_{a}$ of the 2-current of the knot $\gamma$. If the knot $\gamma$ is homologically trivial, one can find a representative of the class $\eta_{\gamma}$ with $\Lambda_{\gamma}^{a b}=0$ and $N_{\gamma}^{a b c}=0$, in agreement with equation (2.10). Whereas when the knot $\gamma$ does not represent the trivial element of $H_{1}(M)$, the components $\Lambda_{\gamma}^{a b}$ and $N_{\gamma}^{a b c}$ are nontrivial.

Any class $A \in H_{D}^{1}(M)^{*}$, which is associated with the $U(1)$ principal bundle that is labelled by $\gamma \in H_{1}(M)$, can be written [5] as

$$
A=\widehat{A}_{\gamma}+\omega=\eta_{\gamma}+\omega
$$

with $\omega \in \Omega_{\mathbb{Z}}^{2}(M)^{*}$. As already stated after equation (2.12), instead of considering directly distributional configurations, one could start with a smooth DB class $\widehat{A}_{\gamma} \in H_{D}^{1}(M)$ and then 
take the distributional $\widehat{A}_{\gamma} \rightarrow \eta_{\gamma}$ limit; in both cases one finds the same results. One has

$$
\int A * A=\int\left(\omega * \omega+2 \omega * \eta_{\gamma}+\eta_{\gamma} * \eta_{\gamma}\right)
$$

In order to give a well defined meaning to $\int \eta_{\gamma} * \eta_{\gamma}$, one can introduce a framing for $\gamma$ (the specific procedure will be discussed in Section 3.2). For now we note that, as a consequence of the definition [5] of the framing method, the introduction of a framing for $\gamma$ has the effect of trivializing (from the Deligne-Beilinson point of view) the star product $\eta_{\gamma} * \eta_{\gamma}$, that is

$$
\int \eta_{\gamma} * \eta_{\gamma}=0 \quad \bmod \mathbb{Z}
$$

Therefore, for framed $\gamma$ one finds

$$
\frac{\int D \omega e^{i S\left[\widehat{A}_{\gamma}+\omega\right]}}{\int D \omega e^{i S[\omega]}}=\frac{\int D \omega e^{i 2 \pi k \int \omega * \omega} e^{i 4 \pi k \int \omega * \eta_{\gamma}}}{\int D \omega e^{i S[\omega]}} .
$$

Let us consider the integral $\int \omega * \eta_{\gamma}$; since $\omega \in \Omega_{\mathbb{Z}}^{2}(M)^{*}$, a representative of the product $\omega * \eta_{\gamma}$ has Čech-de Rham structure

$$
\omega * \eta_{\gamma} \leftrightarrow\left(\omega^{a} \wedge d V_{\gamma}^{a}, 0, \cdots, 0\right)
$$

which does not depend on the nontrivial components $\Lambda_{\gamma}^{a b}$ and $N_{\gamma}^{a b c}$ of the decomposition (3.1) of $\eta_{\gamma}$. The crucial point now is that, for each element $\gamma$ of the torsion group $T(M)$, one can find a representative 1-current $\alpha_{\gamma}$ that can be used - in the integral $\int \omega * \eta_{\gamma}$ - in the place of $\eta_{\gamma}$, i.e. $\forall \omega$

$$
\int \omega * \eta_{\gamma}=\int \omega \wedge d \alpha_{\gamma} \quad \bmod \mathbb{Z}
$$

Indeed, since $\gamma$ is a torsion knot, one can find a nonvanishing integer $p_{\gamma}$ such that $p_{\gamma} \gamma=$ $\Gamma=\partial \Sigma$. Consequently at the level of currents

$$
\alpha_{\gamma}=\frac{1}{p_{\gamma}} \alpha_{\Sigma}
$$

where $\alpha_{\Sigma}$ is the 1-current of the surface $\Sigma$. Let $\widetilde{\alpha}_{\gamma}$ be the class which is associated with $\alpha_{\gamma}$,

$$
\widetilde{\alpha}_{\gamma} \leftrightarrow\left(\frac{1}{p_{\gamma}} \alpha_{\Sigma}^{a}, 0,0\right)
$$

then, by taking into account equation (3.6), one has

$$
\int \omega * \eta_{\gamma}=\int \omega \wedge d \alpha_{\gamma}=\int \omega * \widetilde{\alpha}_{\gamma} \quad \bmod \mathbb{Z} .
$$


Thus one can compute the path-integral (3.5) by using the method that has been described in Section 2. From equations (2.8) and (2.12) it follows that

$$
\begin{aligned}
\frac{\int D \omega e^{i S\left[\widehat{A}_{\gamma}+\omega\right]}}{\int D \omega e^{i S[\omega]}} & =\left\langle\left\langle e^{4 \pi i k \int \omega * \widetilde{\alpha}_{\gamma}}\right\rangle\right\rangle=e^{-2 \pi i k \int \widetilde{\alpha}_{\gamma} * \widetilde{\alpha}_{\gamma}} \\
& =e^{-2 \pi i\left(k / p_{\gamma}^{2}\right) \int \alpha_{\Sigma} \wedge d \alpha_{\Sigma}}=e^{-2 \pi i k N_{\Gamma} / p_{\gamma}^{2}}
\end{aligned}
$$

where the integer $N_{\Gamma}$ denotes the self-linking number of $\Gamma$ in $M$

$$
N_{\Gamma}=\left.\ell k\left(\Gamma, \Gamma_{\mathrm{f}}\right)\right|_{M}
$$

The self-linking number (3.12) is well defined because $\Gamma$ is homologically trivial and, as it is shown in the following section, one can actually choose $\Gamma$ to be a unknot inside a 3 -ball $\mathcal{B}$ in the 3-manifold $M$.

Definition 3.1. Let us introduce the $D B$ class $A_{\gamma}^{0}$ by means of the definition

$$
A_{\gamma}^{0}=\eta_{\gamma}-\widetilde{\alpha}_{\gamma}
$$

From equations (3.1)-(3.10) it follows that a representative of the class $A_{\gamma}^{0}$ can be described by the Čech-de Rham components

$$
A_{\gamma}^{0} \leftrightarrow\left(0, \widetilde{\Lambda}_{\gamma}^{a b}, N_{\gamma}^{a b c}\right)
$$

Since the first component of the representation (3.14) is vanishing, $A_{\gamma}^{0}$ corresponds to the gauge orbit of a flat connection. Equation (3.10) implies that, for any $\omega \in \Omega_{\mathbb{Z}}^{2}(M)^{*}$, one has

$$
\int \omega * A_{\gamma}^{0}=0 \quad \bmod \mathbb{Z}
$$

Hence if, instead of $\eta_{\gamma}$, one takes $A_{\gamma}^{0}$ as origin of the fibre over $\gamma \in H_{1}(M)$, decomposition (3.2) reads

$$
A=A_{\gamma}^{0}+\omega
$$

with $\omega \in \Omega_{\mathbb{Z}}^{2}(M)^{*}$, and one finds

$$
\int A * A=\int A_{\gamma}^{0} * A_{\gamma}^{0}+\int \omega * \omega \quad \bmod \mathbb{Z} .
$$


Consequently the path-integral (3.11), that does not depend on the choice of the origin in the space of gauge orbits, becomes

$$
\frac{\int D \omega e^{i S\left[\widehat{A}_{\gamma}+\omega\right]}}{\int D \omega e^{i S[\omega]}}=\frac{\int D \omega e^{i S\left[A_{\gamma}^{0}+\omega\right]}}{\int D \omega e^{i S[\omega]}}=e^{i S\left[A_{\gamma}^{0}\right]} \frac{\int D \omega e^{i S[\omega]}}{\int D \omega e^{i S[\omega]}}=e^{i S\left[A_{\gamma}^{0}\right]} .
$$

This concludes the proof of Proposition 1.

Equations (3.11) and (3.18) imply

$$
e^{i S\left[A_{\gamma}^{0}\right]}=e^{-2 \pi i k N_{\Gamma} / p_{\gamma}^{2}} .
$$

In order to evaluate the amplitude $e^{i S\left[A_{\gamma}^{0}\right]}=e^{-2 \pi i k N_{\Gamma} / p_{\gamma}^{2}}$ for each $\gamma \in T(M)$ and produce the expression of the quadratic form on the torsion group, it is convenient to introduce a surgery presentation of $M$.

Before proceeding with the path-integral computation of $e^{i S\left[A_{\gamma}^{0}\right]}$, let us point out the main features of the two different choices $-\eta_{\gamma}$ and $A_{\gamma}^{0}-$ of the origins for the fibres of the DB affine bundle $H_{D}^{1}(M)^{*}$. The following $\mathbb{R} / \mathbb{Z}$-valued integrals clearly display the basic peculiarities of $\eta_{\gamma}$ and $A_{\gamma}^{0}$,

$$
\begin{array}{cc}
\int \eta_{\gamma} * \eta_{\gamma}=0, & \int \omega * \eta_{\gamma} \neq 0 \\
\int A_{\gamma}^{0} * A_{\gamma}^{0} \neq 0, & \int \omega * A_{\gamma}^{0}=0 .
\end{array}
$$

For the class $A_{\gamma}^{0}$ one can find smooth representatives, whereas $\eta_{\gamma}$ is in essence distributional; indeed $\eta_{\gamma}$ has precisely been chosen in order to trivialize, with a framed knot $\gamma$, the star product $\eta_{\gamma} * \eta_{\gamma}$. Differently from $\eta_{\gamma}$, the class $A_{\gamma}^{0}$ can be taken as origin for $H_{D}^{1}(M)^{*}$ and for $H_{D}^{1}(M)$ as well. Because $H^{1}(M)=0$, for each $\gamma \in H_{1}(M)=T(M), A_{\gamma}^{0}$ represents a canonical origin for $H_{D}^{1}$ exactly as the zero or vanishing class $\widehat{A}_{0}=0$ can be regarded as the canonical origin for the fibre over the element $0 \in H_{1}(M) \simeq H^{2}(M)$. Let us recall that such a canonical origin $A_{\gamma}^{0}$ does not exist [5] on the fibres over the freely generated component $F(M)$ of $H_{1}(M)$.

From the knowledge of the Cech-de Rham representation (3.14), one could in principle compute the amplitude $e^{i S\left[A_{\gamma}^{0}\right]}$ directly by using the relation

$$
\int A_{\gamma}^{0} * A_{\gamma}^{0} \overline{\overline{\mathbb{Z}}}\left\langle N_{\gamma}^{2} \odot \widetilde{\Lambda}_{\gamma}^{1}, \xi_{3}^{0}\right\rangle
$$

where $\widetilde{\Lambda}_{\gamma}^{1}$ and $N_{\gamma}^{2}$ denote the collections $\left\{\widetilde{\Lambda}_{\gamma}^{a b}\right\}$ and $\left\{N_{\gamma}^{a b c}\right\}$ respectively, $\xi_{3}^{0}$ denotes the points generated by a polyhedral decomposition of the manifold $M$, the pairing $\langle$,$\rangle coincides with$ 
Cech chain-cochain pairing and the product $\odot$ is precisely defined in Ref.[12]. Since $\Gamma=p_{\gamma} \gamma$ is homologically trivial, one has $p_{\gamma} N_{\gamma}^{2}=\delta \Xi_{\Gamma}^{1}$ where $\delta$ denotes the Čech coboundary operator. Then [15]

$$
\int A_{\gamma}^{0} * A_{\gamma}^{0} \overline{\overline{\mathbb{Z}}}-\frac{1}{p_{\gamma}}\left\langle\Xi_{\Gamma}^{1}, \tau_{1}^{\gamma}\right\rangle,
$$

in which $\tau_{1}^{\gamma}$ represents a torsion cycle homologous to $\gamma$ in the Čech formalism. In particular, if the good covering $\left\{\mathcal{U}_{a}\right\}$ of $M$ is also a good cover of $\gamma$, the polyhedral decomposition of $M$ can be chosen to give also a polyhedral decomposition of $\gamma$ in such a way that the collection $\tau_{1}^{\gamma}$ is just the Čech cycle generated by this decomposition. Finally, $\left\langle\Xi_{\Gamma}^{1}, \tau_{1}^{\gamma}\right\rangle$ is the Čech equivalent of the intersection $\Gamma \cap c$ where $c$ is a singular chain such that $\Gamma=b c$. These intersections $[17,18]$ precisely define the torsion quadratic form $Q$.

\subsection{Surgery presentation}

Each 3-manifold $M$ admits a integer surgery presentation in $S^{3}$; let the surgery instruction be described by the framed link $\mathcal{L}=\mathcal{L}_{1} \cup \mathcal{L}_{2} \cdots \cup \mathcal{L}_{m} \subset S^{3}$. The integer surgery coefficients $\left\{a_{t}\right\}$ (with $t=1,2, \ldots, m$ ) coincide with the self-linking numbers of the link components, i.e. $\left\{a_{t}\right\}$ correspond to the diagonal elements of the linking matrix $\mathbb{L}$. With the introduction of a orientation for $\mathcal{L}$, the linking matrix elements are given by $\mathbb{L}_{t s}=\ell k\left(\mathcal{L}_{t}, \mathcal{L}_{s \mathrm{f}}\right)$ where $\mathcal{L}_{s \mathrm{f}}$ denotes the framing of $\mathcal{L}_{s}$. Let the homology group of $S^{3}-\mathcal{L}$ be generated by $\left\{G_{1}, \ldots, G_{m}\right\}$, each generator $G_{t}$ is a small circle linked with $\mathcal{L}_{t}$ and oriented in such a way that $\ell k\left(G_{t}, \mathcal{L}_{t}\right)=1$. The group $H_{1}(M)$ has the presentation shown in equation (1.10); a generic element $\gamma \in H_{1}(M)$ can be written as

$$
\gamma=\sum_{i=1}^{w} n_{i} h_{i}
$$

where the generators $\left\{h_{i}\right\}$ (with $i=1,2, \ldots, w$ ) of $H_{1}(M)$ can be expressed in terms of $\left\{G_{1}, \ldots, G_{m}\right\}$ as shown in equation (1.15) and each integer $n_{i}$ takes values in the residue class of integers modulo the torsion number $p_{i}$, i.e. $n_{i}=0,1,2, \ldots, p_{i}-1$. The sum over the elements of $H_{1}(M)$ corresponds to a multiple sum over the integer coefficients $\left\{n_{i}\right\}$. Equations (3.23) and (1.15) give

$$
\gamma=\sum_{i=1}^{w} \sum_{t=1}^{m} n_{i} B_{i t} G_{t}
$$

In the surgery presentation of a generic 3-manifold $M$, the ambient isotopy classes of (framed) links in $M$ are described by links in the complement of the surgery link $\mathcal{L}$ in $S^{3}$. In 
particular, the knot that represents the element $\gamma \in H_{1}(M)$ is described by a framed knot $\gamma \subset S^{3}-\mathcal{L}$.

Let us introduce a correspondence between the composition law for the elements of $H_{1}(M)$ and a composition law for the framed knots in $S^{3}-\mathcal{L}$ that represent these elements.

Definition 3.2. If - as group elements - $\gamma=\gamma_{1}+\gamma_{2}$ where $\gamma_{1} \neq \gamma_{2}$, then the corresponding framed knots are related as

$$
\gamma=\gamma_{1} \# \gamma_{2}
$$

The group relation $\gamma=\gamma_{1}-\gamma_{2}$ corresponds to $\gamma=\gamma_{1} \#\left(-\gamma_{2}\right)$. The group relation $2 \gamma=\gamma+\gamma$ corresponds to the band connected sum of a knot with itself which is defined to be

$$
2 \gamma=\gamma \# \gamma_{\mathrm{f}}
$$

where $\gamma_{\mathrm{f}}$ denotes the framing of $\gamma$.

Relations (3.25) and (3.26) are consistent with the abelian composition law of the homology group; only the framing choice of the knots needs to be discussed. From the group relation $0=\gamma-\gamma$ and equation (2.14) it follows that any knot that represents the zero element of $H_{1}(M)$ must have trivial framing with respect to the sphere $S^{3}$ of the surgery presentation. For each nontrivial torsion knot $\gamma$, one can find an integer $p_{\gamma}>1$ such that the knot $\Gamma=p_{\gamma} \gamma$ - which is the sum of $p_{\gamma}$ copies of $\gamma$ - is homologically trivial; therefore, $\gamma$ also must have trivial framing with respect to $S^{3}$. To sum up, the consistency of Definition 3.2 requires that all the framed knots that represent the elements of $H_{1}(M)$ must have trivial framing with respect to the sphere of the surgery presentation.

The triviality condition — with respect to surgery $S^{3}$ - for the framings of the knots that represents the elements of $H_{1}(M)$ can also be obtained in the path-integral derivation of the surgery rules for the expectation values of the link holonomies; this issue will be discussed in a forthcoming article [22].

According to the Definition 3.2, the group relation (3.24) can be interpreted in terms of sum of knots; each framed knot $\gamma$ that represents an element of the homology group can be obtained from the framed knots $\left\{G_{1}, \ldots, G_{m}\right\}$ by means of a finite sequence of band connected sum operations. In order to obtain trivial framings for all the $\gamma$ knots, we shall choose the framings $\left\{G_{1 \mathrm{f}}, \ldots, G_{m \mathrm{f}}\right\}$ of $\left\{G_{1}, \ldots, G_{m}\right\}$ to be trivial with respect to the sphere $S^{3}$ of the surgery presentation

$$
\left.\ell k\left(G_{t}, G_{t \mathrm{f}}\right)\right|_{S^{3}}=0 \quad, \quad \forall t=1,2, . ., m
$$




\subsection{Linking numbers}

The linking number $\left.\ell k\left(C_{1}, C_{2}\right)\right|_{S^{3}}$ of two knots $C_{1} \subset S^{3}-\mathcal{L}$ and $C_{2} \subset S^{3}-\mathcal{L}$ - computed with respect to $S^{3}$ - does not necessarily coincide with the linking number $\left.\ell k\left(C_{1}, C_{2}\right)\right|_{M}$ of $C_{1}$ and $C_{2}$ which is possibly defined in $M$. Let $\mathcal{L}_{t}$ (with fixed $t$ ) be one component of the surgery link $\mathcal{L}$ with integer surgery coefficient $a_{t}$. In the surgery construction of $M$, the interior $\stackrel{\circ}{V}$ of a tubular neighborhood $V$ of $\mathcal{L}_{t}$ is removed from $S^{3}$; then $V$ is sewed with $S^{3}-\stackrel{\circ}{V}$ by means of a boundary gluing homeomorphism $h: \partial V \rightarrow \partial\left(S^{3}-\stackrel{\circ}{V}\right)$. The framing $\mathcal{L}_{t \mathrm{f}}$ of $\mathcal{L}_{t}$ is isotopic in $S^{3}-\mathcal{L}$ with the image $h(\mu)$ of the meridian $\mu$ of the solid torus $V \subset M$. Now the meridian $\mu \subset V \subset M$ has a canonical framing $\mu_{\mathrm{f}} \subset V \subset M$ which belongs to the boundary $\partial V ; \mu_{\mathrm{f}}$ also represents a possible meridian for the solid torus $V$ and the two meridians $\mu$ and $\mu_{\mathrm{f}}$ are parallel on the surface $\partial V$. The $\operatorname{knot} h\left(\mu_{\mathrm{f}}\right) \subset S^{3}-\mathcal{L}$ represents a possible framing for $h(\mu)$. Since $h(\mu)$ is ambient isotopic with $\mathcal{L}_{t \mathrm{f}}$, the knot $h\left(\mu_{\mathrm{f}}\right)$ also defines a framing $\mathcal{L}_{t \mathrm{f}}^{\mathrm{f}}$ for $\mathcal{L}_{t \mathrm{f}}$ that will be called the canonical framing of $\mathcal{L}_{t \mathrm{f}}$. Both $h(\mu)$ and $h\left(\mu_{\mathrm{f}}\right)$ belong to the boundary torus $\partial\left(S^{3}-\stackrel{\circ}{V}\right)$ and, according to the surgery instructions, the linking number of $h(\mu)$ and $h\left(\mu_{\mathrm{f}}\right)$ is equal to $a_{t}$ with respect to the sphere $S^{3}$ of the surgery presentation

$$
\left.\ell k\left(\mathcal{L}_{t \mathrm{f}}, \mathcal{L}_{t \mathrm{f}}^{\mathrm{f}}\right)\right|_{S^{3}}=\left.\ell k\left(h(\mu), h\left(\mu_{\mathrm{f}}\right)\right)\right|_{S^{3}}=a_{t} .
$$

In the 3-manifold $M$, the knots $h(\mu)$ and $h\left(\mu_{\mathrm{f}}\right)$ are ambient isotopic - by constructionwith $\mu$ and $\mu_{\mathrm{f}}$ respectively. Any meridinal disc of $V$ whose boundary is $\mu$ does not intersect $\mu_{\mathrm{f}}$. Therefore if $\mu$ and $\mu_{\mathrm{f}}$ are transported inside $V \subset M, \mu$ and $\mu_{\mathrm{f}}$ are ambient isotopic in $M$ with two untied unknots which belong to a 3-ball $\mathcal{B} \subset M$ and which are unlinked in the interior of $\mathcal{B}$. Consequently one has

$$
\left.\ell k\left(\mathcal{L}_{t \mathrm{f}}, \mathcal{L}_{t \mathrm{f}}^{\mathrm{f}}\right)\right|_{M}=\left.\ell k\left(h(\mu), h\left(\mu_{\mathrm{f}}\right)\right)\right|_{M}=\left.\ell k\left(\mu, \mu_{\mathrm{f}}\right)\right|_{M}=0
$$

The property which is encoded in equations (3.28) and (3.29) can also be expressed in the following convenient form.

Suppose that a given framed knot in $M$ is described - in the surgery presentation - by a framed knot $C \subset S^{3}-\mathcal{L}$ with framing $C_{\mathrm{f}}$ such that

$$
\left.\ell k\left(C, C_{\mathrm{f}}\right)\right|_{S^{3}}=0
$$

Let us assume that, in the sphere of the surgery presentation, $C$ is ambient isotopic - as an unframed knot - with the knot $\mathcal{L}_{t \mathrm{f}}$; in compact notations, this is denoted by $C \sim \mathcal{L}_{t \mathrm{f}}$. Then one has

$$
\left.\ell k\left(C, C_{\mathrm{f}}\right)\right|_{M}=-\left.\ell k\left(\mathcal{L}_{t \mathrm{f}}, \mathcal{L}_{t \mathrm{f}}^{\mathrm{f}}\right)\right|_{S^{3}}=-a_{t}
$$


Indeed, under the action of $a_{t}$ right-handed twist homeomorphisms of a tubular neighborhoods of $C$, equations (3.30) is transformed into equation (3.28) and equation (3.31) becomes equation (3.29).

In order to generalize equations (3.28) and (3.29), let us consider the sum $\mathcal{P}=\mathcal{L}_{t \mathrm{f}} \# \mathcal{L}_{s f}$, with for instance $t \neq s$. In agreement with equation (2.13), the canonical framing $\mathcal{P}_{\mathrm{f}}$ of $\mathcal{P}$ satisfies [19]

$$
\left.\ell k\left(\mathcal{P}, \mathcal{P}_{\mathrm{f}}\right)\right|_{S^{3}}=a_{t}+a_{s}+2 \ell k\left(\mathcal{L}_{t}, \mathcal{L}_{s}\right)
$$

According to the surgery construction, the knot $\mathcal{P} \subset S^{3}-\mathcal{L}$ represents a framed unknot which belongs to a 3 -ball in $M$ and

$$
\left.\ell k\left(\mathcal{P}, \mathcal{P}_{\mathrm{f}}\right)\right|_{M}=0
$$

Therefore equations (3.30) and (3.31) admit the following generalization.

Lemma 3.1. Let $C \subset S^{3}-\mathcal{L}$ be a framed knot with framing $C_{\mathrm{f}}$ such that

$$
\left.\ell k\left(C, C_{\mathrm{f}}\right)\right|_{S^{3}}=0 .
$$

Suppose that $C \sim \mathcal{L}^{\#}$, i.e. $C$ is ambient isotopic - as a unframed knot-with the knot $\mathcal{L}^{\#} \subset S^{3}-\mathcal{L}$, in which $\mathcal{L}^{\#}$ corresponds to a finite sequence of band connected sums of the framed surgery link components $\left\{\mathcal{L}_{t \mathrm{f}}\right\}$. Let $\mathcal{L}_{\mathrm{f}}^{\#}$ be the canonical framing of $\mathcal{L}^{\#}$; then $C$ represents [21] a framed unknot in $M$ that belongs to a 3-ball inside the 3-manifold $M$ with self-linking number

$$
\left.\ell k\left(C, C_{\mathrm{f}}\right)\right|_{M}=-\left.\ell k\left(\mathcal{L}^{\#}, \mathcal{L}_{\mathrm{f}}^{\#}\right)\right|_{S^{3}} .
$$

\subsection{The quadratic form}

Since a generic element $\gamma$ of $H_{1}(M)$ can be written as a linear combination (3.24) of the generators $\left\{G_{t}\right\}$, the element $\Gamma=p_{\gamma} \gamma$ reads

$$
\Gamma=p_{\gamma} \gamma=\sum_{i=1}^{w} \sum_{t=1}^{m} n_{i} B_{i t} p_{\gamma} G_{t}
$$

In agreement with the Definition 3.2 and relation (3.36), the framed knot $\Gamma$ can be understood as a band connected sum of the knots $\left\{G_{t}\right\}$. Since each knot $G_{t}$ has trivial framing in $S^{3}$, the knot $\Gamma$ also has trivial framing in $S^{3}$,

$$
\left.\ell k\left(\Gamma, \Gamma_{\mathrm{f}}\right)\right|_{S^{3}}=0
$$


As $\Gamma$ is homologically trivial, the knot $\Gamma$ is ambient isotopic — as an unframed knot - with a knot $\Gamma^{\#}$ in $S^{3}-\mathcal{L}$ which is a band connected sum of the surgery link framing components $\left\{\mathcal{L}_{t \mathrm{f}}\right\}$. Therefore, in order to find the self-linking number of $\Gamma$ in $M$, one can use the analogue of equations (3.34) and (3.35). We only need to determine $\Gamma^{\#}$.

The homology decomposition (1.14) gives rise to the following relation

$$
\mathcal{L}_{t \mathrm{f}} \sim \mathcal{L}_{t \mathrm{f}}^{\#}=\sum_{s=1}^{m} \mathbb{L}_{t s} G_{s}
$$

i.e. in the sphere $S^{3}$ the knot $\mathcal{L}_{t \mathrm{f}}$ is ambient isotopic - as an unframed knot- with the knot $\mathcal{L}_{t \mathrm{f}}^{\#}$ which coincides with the band connected sum $\sum_{s=1}^{m} \mathbb{L}_{t s} G_{s}$. The inverse $\mathbb{L}^{-1}$ of the linking matrix in $\mathbb{R}^{m}$ has rational matrix elements, this means that

$$
\mathbb{L}_{t s}^{-1}=\frac{d_{t s}}{p}
$$

where $d_{s t} \in \mathbb{Z}$ and $p=\operatorname{Det} \mathbb{L}=p_{1} p_{2} \cdots p_{w}>0$, where the torsion numbers $\left\{p_{i}\right\}$ are the integers defined in equation (1.8). Therefore, for any $\gamma$ one can choose $p_{\gamma}=p$. Relation (3.38) can also be expressed as

$$
p G_{t} \sim\left(p G_{t}\right)^{\#}=\sum_{s=1}^{m}\left(p \mathbb{L}_{t s}^{-1}\right) \mathcal{L}_{s \mathrm{f}}=\sum_{s=1}^{m} d_{t s} \mathcal{L}_{s \mathrm{f}} .
$$

Consequently, with the choice $p_{\gamma}=p$, from equations (3.36) and (3.40) it follows that

$$
\Gamma \sim \Gamma^{\#}=\sum_{i=1}^{w} \sum_{t=1}^{m} \sum_{s=1}^{m} n_{i} B_{i t} d_{t s} \mathcal{L}_{s f},
$$

where $n_{i} B_{i t} d_{t s}$ are integers. By using equations (2.13) and (2.14) recursively, one finds that the canonical framing $\Gamma_{\mathrm{f}}^{\#}$ of $\Gamma^{\#}$ is determined [21] by

$$
\begin{aligned}
\left.\ell k\left(\Gamma^{\#}, \Gamma_{\mathrm{f}}^{\#}\right)\right|_{S^{3}} & =\sum_{i=1}^{w} \sum_{t=1}^{m} \sum_{s=1}^{m} \sum_{j=1}^{w} \sum_{r=1}^{m} \sum_{u=1}^{m} n_{i} B_{i t} d_{t s} n_{j} B_{j r} d_{r u} \mathbb{L}_{s u} \\
& =p^{2} \sum_{i=1}^{w} \sum_{j=1}^{w} n_{i} n_{j}\left(\sum_{t=1}^{m} \sum_{s=1}^{m} B_{i t} B_{j s} \mathbb{L}_{t s}^{-1}\right) .
\end{aligned}
$$

Therefore, according to equation (3.35) of Lemma 3.1, one has

$$
N_{\Gamma}=\left.\ell k\left(\Gamma, \Gamma_{\mathrm{f}}\right)\right|_{M}=-\left.\ell k\left(\Gamma^{\#}, \Gamma_{\mathrm{f}}^{\#}\right)\right|_{S^{3}}=-p^{2} \sum_{i=1}^{w} \sum_{j=1}^{w} n_{i} n_{j} Q_{i j}
$$


where $Q_{i j}$ is shown in equation (1.17)

$$
Q_{i j}=\sum_{t, s=1}^{m} B_{i t} B_{j s} \mathbb{L}_{t s}^{-1}
$$

Then equation (3.19) takes the form

$$
e^{i S\left[A_{\gamma}^{0}\right]}=e^{2 \pi i k \sum_{i j} n_{i} n_{j} Q_{i j}}
$$

which coincides with equation (1.13). Finally, according to the definition (1.4), the explicit path-integral computation of the normalized partition function of the $U(1)$ Chern-Simons theory gives

$$
Z_{k}(M)=\sum_{n_{1}=0}^{p_{1}-1} \sum_{n_{2}=0}^{p_{2}-1} \cdots \sum_{n_{w}=0}^{p_{w}-1} e^{2 \pi i k \sum_{i j} n_{i} n_{j} Q_{i j}}
$$

This concludes the derivation of expression (1.17).

The Reshetikhin-Turaev $U(1)$ surgery invariant $I_{k}(M)$ of the 3 -manifold $M$, which admits a surgery presentation in $S^{3}$ with surgery link $\mathcal{L}$, is defined by $[7,8,9,23]$

$$
I_{k}(M)=(2 k)^{-m / 2} e^{i \pi \sigma(\mathcal{L}) / 4} \sum_{q_{1}=1}^{2 k} \cdots \sum_{q_{m}=1}^{2 k} e^{-(2 \pi i / 4 k) \sum_{i j=1}^{m} q_{i} q_{j} \mathbb{L}_{i j}}
$$

where $\sigma(\mathcal{L})$ denotes the signature of the linking matrix $\mathbb{L}$ which is associated with the surgery link $\mathcal{L}$ (which has $m$ components). The multiple sum — which appears in expression (3.46)can be transformed by means of the Deloup-Turaev reciprocity formula [24], which represents a generalization of the reciprocity formula [25] for Gauss sums. The symmetric bilinear form on the lattice $W$ of Theorem 1 of the article [24] corresponds to the bilinear form which is defined by the linking matrix $\mathbb{L}$, and the sum over the elements in the dual lattice $W^{\bullet}$ is represented - in our case - by the sum over the elements of the homology group $H_{1}(M)$. According to equation (3) of Ref. [24], one has

$$
Z_{k}(M)=\left(p_{1} p_{2} \cdots p_{w}\right)^{1 / 2} I_{k}(M)
$$

In the particular example illustrated in the next section, the validity of the above relation will also be verified by a direct application of the standard [25] reciprocity formula for Gauss sums. 


\section{One example}

Let us consider as illustrative example the closed oriented 3-manifold $M_{2,6}$ that corresponds to the surgery link $\mathcal{L} \subset S^{3}$ shown in Figure 3.1.

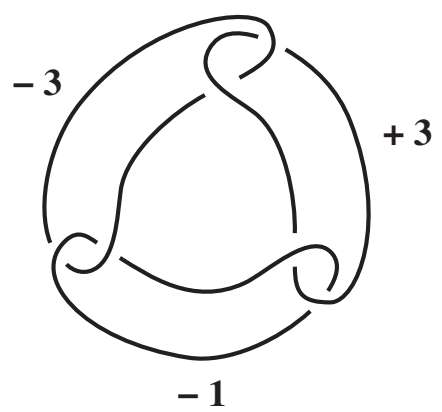

Figure 3.1. Surgery presentation in $S^{3}$ of the manifold $M_{2,6}$.

The values of the surgery coefficients, which are associated with the three link components $\mathcal{L}=\mathcal{L}_{1} \cup \mathcal{L}_{2} \cup \mathcal{L}_{3}$, are $-3,+3$ and -1 respectively. One can introduce a orientation for $\mathcal{L}$ so that the linking matrix is given by

$$
\mathbb{L}=\left(\begin{array}{ccc}
-3 & 1 & 1 \\
1 & 3 & 1 \\
1 & 1 & -1
\end{array}\right) .
$$

Let $\left\{G_{1}, G_{2}, G_{3}\right\}$ be the generators of the homology group of $S^{3}-\mathcal{L} ; G_{i}$ (for $i=1,2,3$ ) is a small circle linked with $\mathcal{L}_{i}$ and oriented in such a way that $\ell k\left(G_{i}, \mathcal{L}_{i}\right)=+1$. The generators $G_{1}, G_{2}$ and $G_{3}$ have trivial framing with respect to the sphere $S^{3}$ of the surgery presentation,

$$
\left.\ell k\left(G_{t}, G_{t \mathrm{f}}\right)\right|_{S^{3}}=0 \quad, \quad \text { for } \quad=1,2,3 .
$$

The three conditions $\sum_{j} \mathbb{L}_{i j} G_{j}=0$ (for $\left.i=1,2,3\right)$ that specify $H_{1}\left(M_{2,6}\right)$ can be expressed in the form

$$
\begin{aligned}
& \text { (a) } G_{3}=G_{1}+G_{2}, \\
& \text { (b) } 6 G_{2}=0, \\
& \text { (c) } 2\left(G_{1}-G_{2}\right)=0 .
\end{aligned}
$$

Therefore $H_{1}\left(M_{2,6}\right)=T\left(M_{2,6}\right)=\mathbb{Z}_{2} \oplus \mathbb{Z}_{6}$ in which one can take

$$
\begin{array}{ll}
h_{1}=G_{1}-G_{2} & \text { as generator for } \mathbb{Z}_{2}, \\
h_{2}=G_{2} & \text { as generator for } \mathbb{Z}_{6} .
\end{array}
$$


Each element $\gamma \in H_{1}\left(M_{2,6}\right)=\mathbb{Z}_{2} \oplus \mathbb{Z}_{6}$ can be written as

$$
\gamma=n_{1} h_{1}+n_{2} h_{2}, \quad \text { with } n_{1}=0,1 \text { and } n_{2}=0,1,2,3,4,5 \text {. }
$$

The inverse of the linking matrix is given by

$$
\mathbb{L}^{-1}=\frac{1}{6}\left(\begin{array}{ccc}
-2 & 1 & -1 \\
1 & 1 & 2 \\
-1 & 2 & -5
\end{array}\right)
$$

and then, in the basis (4.4), one finds

$$
Q=\frac{1}{6}\left(\begin{array}{cc}
-3 & 0 \\
0 & 1
\end{array}\right)
$$

The normalized partition function is equal to

$$
Z_{k}\left(M_{2,6}\right)=\sum_{n_{1}=0}^{1} \sum_{n_{2}=0}^{5} e^{2 \pi i k\left(-3 n_{1}^{2}+n_{2}^{2}\right) / 6}
$$

The Reshetikhin-Turaev invariant $I_{k}\left(M_{2,6}\right)$ is given by

$$
I_{k}\left(M_{2,6}\right)=(2 k)^{-3 / 2} e^{-i \pi / 4} \sum_{q_{1}=0}^{2 k-1} \sum_{q_{2}=0}^{2 k-1} \sum_{q_{3}=0}^{2 k-1} e^{-(2 \pi i / 4 k)\left[-3 q_{1}^{2}+2 q_{1} q_{2}+3 q_{2}^{2}+2 q_{2} q_{3}-q_{3}^{2}+2 q_{3} q_{1}\right]} .
$$

By means of the reciprocity formula [25] for the Gauss sums

$$
\sum_{n=0}^{|c|-1} e^{-\frac{i \pi}{c}\left(a n^{2}+b n\right)}=\sqrt{|c / a|} e^{-\frac{i \pi}{4 a c}\left(|a c|-b^{2}\right)} \sum_{n=0}^{|a|-1} e^{\frac{i \pi}{a}\left(c n^{2}+b n\right)}
$$

which is valid for integers $a, b$ and $c$ such that $a c \neq 0$ and $a c+b=$ even, one obtains

$$
\sum_{q_{3}=0}^{2 k-1} e^{-(2 \pi i / 4 k)\left[-q_{3}^{2}+2 q_{3}\left(q_{2}+q_{1}\right)\right]}=\sqrt{2 k} e^{i \pi / 4} e^{-i(\pi / 2 k)\left(q_{1}+q_{2}\right)^{2}} .
$$

Therefore

$$
I_{k}\left(M_{2,6}\right)=(2 k)^{-1} \sum_{q_{1}=0}^{2 k-1} \sum_{q_{2}=0}^{2 k-1} e^{-(2 \pi i / 4 k)\left[-2 q_{1}^{2}+4 q_{2}^{2}+4 q_{1} q_{2}\right]} .
$$


The reciprocity formula also gives

$$
\sum_{q_{1}=0}^{2 k-1} e^{(i \pi / 2 k)\left[2 q_{1}^{2}-4 q_{1} q_{2}\right]}=\sqrt{\frac{2 k}{2}} e^{i \pi / 4} e^{-i \pi q_{2}^{2} / k} \sum_{n=0}^{1} e^{-i(\pi / 2)\left[2 k n^{2}-4 n q_{2}\right]} .
$$

So one obtains

$$
I_{k}\left(M_{2,6}\right)=\frac{1}{\sqrt{2}}(2 k)^{-1 / 2} e^{i \pi / 4} \sum_{n=0}^{1} e^{-i \pi k n^{2}} \sum_{q_{2}=0}^{2 k-1} e^{-(2 \pi i / 4 k)\left[6 q_{2}^{2}-4 k n q_{2}\right]} .
$$

Again the reciprocity formula produces

$$
\sum_{q_{2}=0}^{2 k-1} e^{-(i \pi / 2 k)\left[6 q_{2}^{2}-4 k n q_{2}\right]}=\frac{\sqrt{2 k}}{\sqrt{6}} e^{-i \pi / 4} e^{i \pi k n^{2} / 3} \sum_{m=1}^{5} e^{i \pi\left(2 k m^{2}-4 k n m\right) / 6}
$$

Therefore

$$
I_{k}\left(M_{2,6}\right)=\frac{1}{\sqrt{12}} \sum_{n=0}^{1} \sum_{m=1}^{5} e^{(2 \pi i k / 6)\left[-2 n^{2}+m^{2}-2 m n\right]}
$$

Let us change variables and put

$$
n=n_{1} \quad, \quad m=n_{2}+n_{1}
$$

with $n_{1}=0,1$ and $n_{2}=0,1,2,3,4,5$. Expression (4.16) finally becomes

$$
I_{k}\left(M_{2,6}\right)=\frac{1}{\sqrt{12}} \sum_{n_{1}=0}^{1} \sum_{n_{2}=1}^{5} e^{2 \pi i k\left(-3 n_{1}^{2}+n_{2}^{2}\right) / 6}
$$

Comparing equations (4.8) and (4.18), one finds

$$
Z_{k}\left(M_{2,6}\right)=(2 \cdot 6)^{1 / 2} I_{k}\left(M_{2,6}\right)
$$

which is in agreement with equation (1.18).

\section{Conclusions}

In the $U(1)$ Chern-Simons theory the Deligne-Beilinson formalism sheds light on the fundamental role played by the flat connections in the computation of the topological invariants. In particular this formalism produces a path-integral derivation of the abelian 
Reshetikhin-Turaev surgery invariant. In a forthcoming article [22] we will show in detail how to compute the link expectation values in a generic 3-manifold by means of the Feynman functional integral; we will take into account both the freely generated component and the torsion component of the homology group.

The Deligne-Beilinson approach to the Chern-Simons field theory also exists [26] in a $(4 n+3)$-dimensional closed manifold. Possible extensions of our results to these higher dimensional cases will be investigated.

Acknowledgments. We wish to thank Riccardo Benedetti for useful discussions.

\section{References}

[1] A.S. Schwarz, Lett. Math. Phys. 2 (1978) 247.

[2] A.S. Schwarz, Commun. Math. Phys. 67 (1979) 1.

[3] C.R. Hagen, Ann. Phys. (N.Y.) 157 (1984) 342.

[4] E. Witten, Commun. Math. Phys. 121 (1989) 351.

[5] E. Guadagnini and F. Thuillier, SIGMA 4 (2008) 078, arXiv:0801.1445.

[6] F. Thuillier, J. Math. Phys. 50, 122301 (2009); arXiv:0901.2485.

[7] N.Y. Reshetikhin and V.G. Turaev, Invent. Math. 103 (1991) 547.

[8] H. Murakami, T. Ohtsuki, and M. Okada, Osaka J. Math. 29 (1992) 545.

[9] F. Deloup, Math. Ann. 319 (2001) 759.

[10] P. Deligne, Théorie de Hodge II, Publ. Math. I.H.E.S. n.40 (1971) 5.

[11] A.A. Beilinson, J. Soviet Math. 30 (1985) 2036.

[12] J.L. Brylinski, Loop spaces, characteristic classes and geometric quantization, Progress in Mathematics, Vol. 107, Birkh?auser Boston, Inc., Boston, MA, 1993.

[13] M. Bauer, G. Girardi, R. Stora and F. Thuillier, JHEP 0508 (2005) 027. 
[14] J. Cheeger and J. Simons, Differential characters and geometric invariants, Stony Brook Preprint 1973; reprinted in Lecture Notes in Mathematics 1167, Geometry and Topology Proc. 1983-84, Eds J. Alexander and J. Harer, Springer 1985.

[15] J.L. Koszul, Travaux de S.S. Chern et J. Simons sur les classes caractéristiques, Séminaire Bourbaki 26ème année, n.440, (1973/74) 69.

[16] M. Kneser and P. Puppe, Math. Zeitschr. 58 (1953) 376.

[17] J. Lannes and F. Latour, Forme quadratique d'enlacement et applications, Astérisque 26 (1975).

[18] A. Gramain, Formes d'intersection et d'enlacement sur une variété, Mémoires de la Société Mathématique de France, 48 (1976), p. 11-19.

[19] D. Rolfsen, Knots and Links, AMS Chelsea Publishing, Providence, 2003.

[20] D. Elworthy and A. Truman, Feynman maps, Cameron-Martin formulae and anharmonic oscillators, Ann. Inst. Henri Poincaré Phys. Théor. 41 (1984) 115.

[21] R. Kirby, Invent. Math. 45 (1978) 35.

[22] E. Guadagnini and F. Thuillier, Path integral and surgery rules in abelian Chern-Simons theory, in preparation.

[23] V.G. Turaev, Quantum invariants of knots and 3-manifolds, de Gruyter Studies in Mathematics 18 (Berlin, 1994).

[24] F. Deloup and V. Turaev, Journal of Pure and Applied Algebra 208 (2007) 153.

[25] C.L. Siegel, Über das quadratische Reziprozitätsgesetz algebraischen Zahlkörpern, Nachr. Acad. Wiss. Göttingen Math. Phys. Kl. 1 (1960) 1.

[26] L. Gallot, E. Pilon and F. Thuillier, Higher dimensional abelian Chern-Simons theories and their link invariants, arXix:1207.1270v1, to appear in J. Math. Phys. 